\title{
Dicer- and BSC-dependent miRNAs during murine anagen hair follicle development
}

Neda Vishlaghi ${ }^{1}$, Thomas S. Lisse ${ }^{1,2^{*}}$

${ }^{1}$ The University of Miami, Department of Biology, Coral Gables, FL 33124

2 Sylvester Comprehensive Cancer Center, Miller School of Medicine, University of Miami, Miami, Florida 33136 USA

${ }^{*}$ Corresponding author

Thomas Lisse Ph.D.

Department of Biology, The University of Miami

tom.lisse@miami.edu

305-284-3957

Keywords: MicroRNA, Dicer, Tarbp2, hair follicle, hair cycle, bulge stem cell, regeneration, anagen

Funding: T.S.L and N.V. are supported by the College of Arts and Sciences, Biology Department, University of Miami, Coral Gables, Florida 


\section{Abstract}

MicroRNAs (miRNAs) are a major class of conserved non-coding RNAs that have a wide range of functions during development and disease. Biogenesis of canonical miRNAs depend on the cytoplasmic processing of pre-miRNAs to mature miRNAs by the Dicer endoribonuclease. Once mature miRNAs are generated, the miRNA-induced silencing complex, or miRISC, incorporates one strand of miRNAs as a template for recognizing complementary target messenger RNAs (mRNAs) to dictate posttranscriptional gene expression. Besides regulating miRNA biogenesis, Dicer is also part of miRISC to assist in activation of the complex. Dicer associates with other regulatory miRISC co-factors such as trans-activation responsive RNA-binding protein (Tarbp2) to regulate miRNA-based RNA interference. Although the functional role of miRNAs within epidermal keratinocytes have been extensively studied within embryonic and post-natal mouse skin, its contribution to the normal function of hair follicle bulge stem cells (BSCs) during post-natal hair follicle development is unknown. With this question in mind, we sought to ascertain whether Dicer-Tarpb2 plays a functional role within BSCs during induced anagen development by utilizing conditional knockout mouse models. Our findings suggest that Dicer, but not Tarbp2, functions within BSCs to regulate induced anagen (growth) development of post-natal hair follicles. These findings strengthen our understanding of miRNA-dependency within hair follicle cells to define a boundary for post-transcriptional gene regulation during anagen development. 


\section{Background}

The functional roles of microRNAs (miRNAs) within epidermal keratinocytes have been extensively studied and entail control over cell proliferation, differentiation, migration, development and epithelial-mesenchymal transitions (Mardaryev et al., 2010, Xu et al., 2011, Yi et al., 2009, Yu et al., 2010). Within embryonic skin of mice, epidermal ablation of core miRNA machinery components Dicer, Ago1/2, Drosha or Dgcr8 disrupts hair follicle (HF) morphogenesis and ensuing hair formation (Andl et al., 2006, Teta et al., 2012, Yi et al., 2006, Yi et al., 2009). Additionally, overexpression studies within keratin 14-positive basal epidermal cells have also shown the inhibitory effects of miR-214 on skin and HF morphogenesis (Ahmed et al., 2014). By and large these findings depict the important role of global miRNAs toward the expansion of HF and skin cells during embryonic and neonatal development.

Only a few studies have investigated the role of miRNAs during the post-natal period within skin cells. For example, Dicer within keratin 5 (K5)-positive epithelial keratinocytes and/or HF epithelial stem cells (Joost S, 2019) of young mice was found to be required for post-natal HF growth and plucking-induced anagen development associated with strong phenotypes (Teta et al., 2012). Recently, miR-218-5p was shown to regulate post-natal skin and HF development by induction of the Wnt signaling pathway (Zhao et al., 2019), however the exact cell types involved remain unclear. Likewise, multiple Dicer-dependent (Lee and Doudna, 2012) miRNAs are expressed in a synchronous pattern following the post-natal hair cycle (Mardaryev et al., 2010, Zhao et al., 2019). Nonetheless, whether miRNAs within post-natal HF bulge stem cells (BSCs) - the main source of telogen to anagen transformation - play a functional role during induced anagen development in mice remains unknown. To help bridge this knowledge gap and advance the field, we conditionally ablated Dicer and one of its regulatory co-factors, trans-activation responsive RNA-binding protein (Tarbp2), specifically within BSCs during induced anagen development. Dicer-Tarbp2 regulates miRNA biogenesis (Pullagura et al., 2018), pre-miRNA cleavage specificity (Lee and 
Doudna, 2012) and facilitates RNA interference by association with the miRNA-induced silencing complex (miRISC) loading complex (Lee et al., 2013). Therefore, we hypothesized that global miRNAs and miRISC-mediated RNA silencing are required within BSCs during induced anagen development.

\section{Results and Discussion}

To test our hypothesis, we performed hair depilation assays during the resting stage of the HF growth cycle (P50) using inducible Tarbp2 and Dicer floxed mouse lines crossed to keratin 15 (K15) PR1Cre transgenic mice (Figure 1A). This system dominantly ablates Tarbp2 and Dicer within outer BSCs upon RU486 treatment and not within cycling and basal interfollicular epidermal cells as previously performed (Joost S, 2019, Teta et al., 2012). By visual inspection, both control Tarbp2 ${ }^{+/}: \mathrm{K} 15 \mathrm{PR} 1 \mathrm{Cre}+$ and experimental Tarbp $2^{\text {floxflox }}:$ K15PR1Cre + mice exhibited hyperpigmentation at 9 days post depilation (DPD) (Figure 1B). Through histological analysis, we observed the expected anagen HFs at 9 DPD among control mice (Figure 1C) and observed no histological nor HF growth (Figure 1D) abnormalities among mutant Tarbp2 $2^{\text {floxflox}: K 15 P R 1 C r e+~ m i c e . ~ W e ~ a l s o ~ a p p l i e d ~ a n ~ u l t i m a t e ~ 3 D ~ i m a g i n g ~ o f ~ s o l v e n t-~}$ cleared organs (UDISCO) whole-mount clearing method and observed no major difference in the extent of hair growth and hair shaft formation after depilation (Figure 1E). In deletion PCR studies, only RU486-treated skin samples derived from Tarbp $2^{\text {floxfllox}}:$ K15PR1Cre+ mice resulted in Tarbp2 deletion (Figure 1F). Collectively, our results suggest that Tarbp2 regulation of miRISC within BSCs is not essential during induced anagen development of HFs.

Since Tarbp2 regulates Dicer activity (Pullagura et al., 2018), we conditionally ablated Dicer within BSCs by generating the Dicer floxflox:K15PR1Cre+ mouse model and monitored anagen development after depilation (Figure 1A). By visual observation, both control Dicer flox/flox:K15PR1Cre- and experimental Dicef floxflox:K15PR1Cre+ mice exhibited hyperpigmentation at 9 DPD (Figure 2A) and similar number of anagen HFs 
(Figure 2B-D), suggesting intact HF stem cell linage specification. However, we observed a small, yet statistically significant decrease in the thickness of skin upon Dicer ablation when compared to controls (Figure 2C), suggesting a mild delay in anagen progression (Paus et al., 1999). Dicer deletion PCR studies confirmed that only RU486-treated skin samples from Dicer mutant mice generated a deletion PCR product (Figure 2E). To further validate Dicer ablation within BSCs, we performed immunofluorescence staining for DICER using an antibody that specifically recognizes exons 22-23 of DICER (i.e. the region of Dicer which is excised). We observed a statistically significant ( $p \leq 0.001$, Student $t$ test) decrease in background normalized DICER immunoreactivity within the BSC compartments of mutant mice when compared to control (Figure 2F). Importantly, we also observed decreased DICER expression within BSC progeny of individual mutant HFs (Figure 2G). In control HFs, we observed DICER-positive cells in the upper ORS, combined with increased presence of DICERpositive cells within the inner root sheath (IRS) and cortex (Cx). The cellular decrease in DICER throughout mutant HFs was likely due to HF resolve to monoclonality as also shown by the K15PR1Cre+:R26R-Confetti reporter line (Figure 2H). Furthermore, the DICER immunostaining pattern in the control animals was consistent with recently available single cell-RNAseq data showing Dicer mRNA expression specifically within a subset of Cx/IRS as well as ORS cells within anagen HFs (Joost S, 2019) (Figure 2I), suggesting the cell types of miRNA dependency. Previous findings have underscored the role of miRNAs during skin development and post-natal skin maintenance by manipulating specific cell types. In summary, we show that miRNAs within K15-postive BSCs help regulate proper post-natal HF anagen development. Furthermore, we show the possibility of co-regulatory redundancy of Tarbp2 by other Dicer associated factors which may help define miRNA specificity within BSCs. Collectively, our findings demonstrate the striking cellular specificity and balance that global Dicer- and BSCdependent miRNAs exhibit during induced anagen development.

\section{Data Availability}


The data supporting the results reported in this article will be provided upon reasonable request.

\section{Conflict of Interest}

The authors state no conflict of interest.

\section{Author Contribution}

N.V. was involved in the formal investigation, methodology, analysis of the data, and the review and editing of the manuscript. T.S.L. was involved in the conceptualization, project administration, formal investigation, methodology, analysis of the data, and writing of the original draft of the manuscript.

\section{Acknowledgement}

We especially thank Legnay Fernandez (U. Miami) and Kathryn Helen Forcone (U. Miami) for their critical comments on this manuscript. We especially thank the Animal Care and Veterinary staff at the Neuroscience Annex (U. Miami) for their animal support.

\section{Figure Legends}

Figure 1. Conditional knock out of Tarbp2 with hair follicle bulge stem cells. A) Schema outlining the genotyping and conditional knockout of Tarbp2 and Dicer within bulge stem cells.

B) Images of the depilated regions of control ( $\left.\operatorname{Tarbp}^{+/+} ; \mathrm{K} 15 \mathrm{PR} 1 \mathrm{Cre}+\right)$ and experimental (Tarbp2 $2^{\text {floxflox; }}$ K15PR1Cre+) mice at 0 days post depilation (DPD) and 9 DPD. C) Histological analysis of 9 DPD skins in both control (Tarbp2 $\left.2^{+/+} ; \mathrm{K} 15 \mathrm{PR} 1 \mathrm{Cre}+\right)$ and experimental ( Tarbp $2^{\text {floxflox }} ; \mathrm{K} 15 \mathrm{PR} 1 \mathrm{Cre}+$ ) mice. Boxed region is magnified in the lower panels. Bars (top panels $=1 \mathrm{~mm}$; bottom panels $=100 \mathrm{um}$ ) 
D) Skin thickness. Distance from the striated muscle layer to the outer epidermal layer $(\mu \mathrm{m})$.

E) Whole mount uDISCO analysis of 9DPD skins in both control

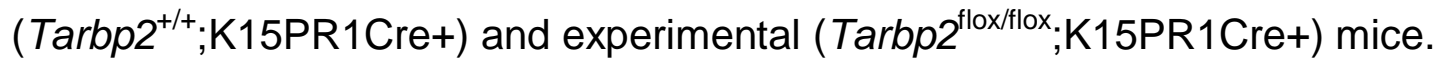

F) Schema of the targeting construct for generation of Tarbp2 floxed mice (top panel). Schema of the PCR strategy to determine Tabp2 ablation efficiency within tissue samples (bottom panel). Right panel shows the Tabp2 deletion PCR results using RU486 pre and post-treated tissue samples.

Figure 2. Conditional knockout of Dicer within hair follicle bulge stem cells. A) Images of the depilated regions of control (Dicer flox/flox;K15PR1Cre-) and experimental (Dicer flox/flox $; K_{15}$ PR1Cre+) mice at 0 days post depilation (DPD) and 9DPD.

B) Histological analysis of 9DPD skins in both control (Dicer flox/flox;K15PR1Cre-) and experimental (Dicer flox/flox;K15PR1Cre+) mice. Boxed region is magnified in the lower panels. Bars (top panels $=1 \mathrm{~mm}$; bottom panels $=100 \mathrm{um}$ )

C) Hair follicle count and skin thickness analysis. Serial skin sections were assessed from control and Dicer cKO mice ( $n=12 ; p \leq 0.001$, Student's t test).

D) Whole-mount uDISCO analysis of 9DPD skins in both control (Dicer $\left.{ }_{\text {flox/flox }} ; \mathrm{K} 15 \mathrm{PR} 1 \mathrm{Cre}-\right)$ and experimental (Dicer $\left.{ }^{\text {flox/flox}} ; \mathrm{K} 15 \mathrm{PR} 1 \mathrm{Cre}+\right)$ mice.

E) Schema of the targeting construct for generation of Dicerfloxed mice. Schema of the PCR strategy to determine Dicer ablation efficiency within tissue samples (top panel). Dicer deletion PCR using RU486 pre- and post-treated tissue samples (bottom panel). F) Immunofluorescence detection of DICER within various hair follicle compartments of control (Dicer $\left.{ }^{+/+} ; \mathrm{K} 15 \mathrm{PR} 1 \mathrm{Cre}+\right)$ and experimental (Dicer flox/flox;K15PR1Cre+) skin. Upper panels depict the individual compartments and cell types that express or do not express DICER. Hf (hair follicle), Sc (sebaceous gland compartment; white dash), Fbs (fibroblasts), Bsc (bulge stem cell compartment; red dash). The lower panels highlight only the Bsc and nuclear staining with DAPI. Bars = 50um. (Right panel) Quantification of DICER expression from immunofluorescence staining. DICER expression was 
analyzed within bulge stem cell compartments ( $n=8 ; p \leq 0.01$, Student's t test) and presented as background normalized mean intensity values.

G) Immunofluorescence detection of DICER within the entire hair follicle of control

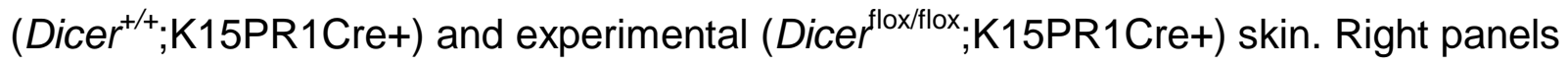
depict 3D-rendered models of DICER expression in control hair follicles. ORS (outer root sheath), IRS (inner root sheath), Cx (cortex). Bars =50um.

H) K15 BSC lineage tracing using the R26R-Confetti:K15PR1Cre+ mouse line.

I) Dicer mRNA expression at the single cell level during hair growth (anagen) and rest (telogen) in full-thickness skin. Permanent epidermis keratinocytes (shown in green) contain subpopulations of resting BSCs and epidermal keratinocytes. The anagen hair follicle keratinocytes (shown in orange) contain subpopulations of cells undergoing growth and differentiation. EPI (permanent epidermal keratinocytes), ANA (anagen hair follicle keratinocytes). EPI cells consist of interfollicular epidermis (IFE) basal cycling $(\mathrm{C})$, basal (B) and suprabasal (SB) cells, upper hair follicle (uHF) basal (B) and suprabasal (SB) cells, sebaceous glands (SG), outer bulge (OB), and hair germ (HG) cells. ANA cells consist of outer layer (OL) cells, germinative layer (GL) cells, inner root sheath (IRS) cells, cortex/cuticle (CX) cells, and medulla (MED) cells.

Data derived from: (Joost S, 2019)

\section{Materials and Methods}

\section{Mouse Models and Breeding}

Dicer- Null conditional (Dicer1 $\left.{ }^{t m 1 B d h} / \mathrm{J}\right)$ mice (referred to as Dicer floxed; JAX stock no. 0063661) and Tarbp2- Null conditional (Tarbp2 $2^{\text {tm1.1Dzw }}$ ) mice (referred to as Tarbp2 $2^{\text {Floxed }}$ ;MGI: 5645258) were received from the Jackson laboratory. The Tg(Krt1-15cre/PGR)22Cot line (referred to as K15-PR1Cre; JAX stock no. 005249) was on a mixed C57BL/6 and SJL background also obtained from Jackson Laboratory and was used for generation of RU486-induced, Cre-mediated gene deletions within BSCs of hair follicles (HFs). Dicer ${ }^{f l o x / f l o x}\left(\operatorname{Dicer}^{f(/ f)}\right)$ and Tarbp2 $2^{f l o x / f l o x}\left(\operatorname{Tarbp}^{f / f f}\right)$ mice were bred with K15-Cre line to achieve cell-specific knockout of targeted regions within BSCs of HFs. 
Lineage tracing was performed using the Gt(ROSA)26Sor ${ }^{\operatorname{tm1}(C A G-B r a i n b o w 2.1) C l e / J}$ mice obtained from the Jackson Laboratory (R26R-Confetti; JAX stock no. 013731). All animals were maintained in a 12-h light (6am to 6pm) and 12-h dark cycle vivarium in the Research Animal Facility at the University of Miami. Animals were provided acidified tap water through filter bottle and irradiated pelleted 2018 Teklad global 18\% protein rodent diet from ENVIGO. In timed pregnancy crosses, pups were dated based on the presence of vaginal plugs and by noting the delivery of newly born pups. All animal studies were approved by the University of Miami Institutional Animal Care and Use Committee (IACUC) protocols.

\section{Genotyping and recombination detection}

All genotyping proceeded by using tail tip excision/partial amputation under the age of 21 days. Dicer floxed allele was genotyped by using primers DicerF1 (CCTGACAGTGACGGTCCAAAG) and DicerR1 (CATGACTCTTCAACTCAAACT). PCR product for genotyping PCR was 420-bp band for Dicer and a 351-bp band for wild type allele. The deletion was genotyped by using primers DicerF1 and DicerDel (CCTGAGCAAGGCAAGTCATTC). PCR product for deletion PCR was a 471-bp band for deletion and a 1300-bp band for the wild type allele. To assay floxed vs. wild type allele in Tarbp2 animals, we used TRS-loxF (CAGAAGCACAGCAGGAACAA) and TRS-loxR (CGTGATATGCACAGCCCACT) primers. PCR product for genotyping PCR was 180-bp band for the floxed and a 130-bp band for the wild type allele. Deletion allele was detected by using primers TRS-loxF and TRS-3loxR (CAAAACCACTTCCCCATGTT). All mice genotyping was based on established protocols listed on the JAX website for each stock animal. PCR program was run according to recommendations: $95^{\circ} \mathrm{C}$ for 5 minutes, and for 35 cycles $95^{\circ} \mathrm{C}$ for 15 seconds, $60^{\circ} \mathrm{C}$ for 15 seconds and $72^{\circ} \mathrm{C}$ for 10 seconds for Dicer genotyping and deletion PCR; $94^{\circ} \mathrm{C}$ for 3 minutes, for 35 cycles $94^{\circ} \mathrm{C}$ for 30 seconds, $61.7^{\circ} \mathrm{C}\left(59^{\circ} \mathrm{C}\right.$ annealing for delete allele) for 30 seconds and $72^{\circ} \mathrm{C}$ for 20 seconds for Tarbp2 PCR.

\section{Conditional knockout studies and hair depilation assay}


Under general anesthesia (inhalation isoflurane), 50-day-old animals were subjected to hair depilation of two separate left and right side of the dorsal area by using Wax Strips. All procedures were performed using sterile instruments and aseptic conditions. Mice received topical daily treatments of $1 \% \mathrm{RU} 486$ in acetone, or acetone only (vehicle), over the depilated area for nine days. Nine days after depilation, animals were sacrificed, and skin samples were collected for analysis.

\section{Ultimate DISCO (UDISCO) whole mount passive clearing technique for skin}

To visualize thick skin tissue and hair morphology, we applied the organic solventbased uDISCO clearing method. Greater than $3 \mathrm{~mm}$-by-3mm skin tissues were fixed in $4 \%$ PFA at $4^{\circ} \mathrm{C}$ overnight. Samples were washed with $0.1 \mathrm{M}$ PBS and subjected to a tert-butanol series (70-100\%; Sigma-Aldrich, 36053) for gradient dehydration for 2-12 hours. Next, dichloromethane (Sigma-Aldrich, 270997) was replaced as a pure solution for the delipidation step for 1 hour at room temperature. A refractive index matching solution was prepared by mixing BABB (benzyl alcohol + benzyl benzoate 1:2; SigmaAldrich, 24122 and W213802) and DPE (diphenyl ether) (Sigma-Aldrich, 240834) at a BABB:DPE ratio of 10:1 (vol/vol). The samples were reacted with the BABB:DPE mixture until they became optically transparent.

\section{Histological, histomorphometric and immunofluorescence analysis}

Skin tissues $\left(1 \times 1 \mathrm{~cm}^{2}\right)$ were dissected from mice and incubated $2-4$ hours in $4 \%$ PFA in PBS ( $\mathrm{pH} 7.4$ ) at $4^{\circ} \mathrm{C}$ and transferred to $70 \% \mathrm{OH}$, before embedding in paraffin wax. $7 \mu \mathrm{m}$-sections were stained with hematoxylin and eosin (H\&E) by The University of Miami Histology Core Services. For immunofluorescence studies, skin tissues $\left(1 \times 1 \mathrm{~cm}^{2}\right)$ were dissected from mice and incubated $2-4$ hours in $4 \%$ PFA in PBS $\left(\mathrm{pH} \mathrm{7.4)}\right.$ at $4^{\circ} \mathrm{C}$ and subjected to graded sucrose treatments (15-30\%) for cryoprotection. These tissues were embedded face down along the midline in OCT embedding medium (Histolab). Cryosections were made using a Leica CM 1850 Cryostat. 10 $\mu \mathrm{m}$-sections were incubated with $0.4 \%$ Triton X-100 in PBS for 30 minutes at RT to reduce background staining. Tissue sections were directly blocked in PBS containing 5\% normal horse serum for 30 minutes at RT. Then incubated with endogenous Mouse IgG blocking 
solution 1:10 in PBS (Unconjugated AffiniPure Fab Fragment Goat Anti-mouse $\lg \mathrm{G}(\mathrm{H}+\mathrm{L})$; Jackson ImmuneResearch Labs, 115-007-003) for 1 hour at RT. Sections were incubated with primary antibodies at optimal dilution for 30 minutes at RT, antibodies used in this study included Dicer (BioLegend. 820201). Following 3x washes for 2 minutes each in PBS-Tween-20, the sections were incubated at room temperature for 20 minutes with corresponding species-specific secondary antibodies (Alexa series, Life Technologies). Following 3x washes in PBS-Tween-20, sections were mounted with Vectashield medium containing DAPI (Vector Laboratories) for nuclei staining. Immunofluorescence microscopy was performed using a Zeiss Observer 7 ApoTome2 unit. Images were captured from BSCs compartment for DICER expression and normalized to acellular dermal regions. Means of expression intensity of individual follicles were compared between control and mutant animals $(n=8)$. Imaris (Bitplane) was used to generate 3D rendered models of Dicer expression. For hair follicle counts (i.e. hair follicles per $2.5 \mathrm{~mm}$ tissue; $n=12$ ) and skin thickness measurements (i.e. distance from panniculus carnosus (muscle) to the outer epidermis; $n=12$ ), serial skin sections were analyzed between control and cKO mice. Two-tailed unpaired T tests were performed between control and cKO data sets using Prism (GraphPad) where the $p$ value summaries were depicted as ${ }^{* * *} p \leq 0.001{ }^{* *} p \leq 0.01$, and ${ }^{*} p \leq 0.05$.

\section{References}

Ahmed MI, Alam M, Emelianov VU, Poterlowicz K, Patel A, Sharov AA, et al. MicroRNA-214 controls skin and hair follicle development by modulating the activity of the Wnt pathway. The Journal of cell biology 2014;207(4):549-67.

Andl T, Murchison EP, Liu F, Zhang Y, Yunta-Gonzalez M, Tobias JW, et al. The miRNA-processing enzyme dicer is essential for the morphogenesis and maintenance of hair follicles. Current biology : $C B$ 2006;16(10):1041-9.

Joost S AK, Jacob T, Sun X, Sivan U, Dalessandri T, Sequeira P, Sandberg R, Kasper M. The molecular anatomy of mouse skin during hair growth and rest. bioRxiv 2019.

Lee HY, Doudna JA. TRBP alters human precursor microRNA processing in vitro. Rna 2012;18(11):2012-9.

Lee HY, Zhou K, Smith AM, Noland CL, Doudna JA. Differential roles of human Dicer-binding proteins TRBP and PACT in small RNA processing. Nucleic acids research 2013;41(13):6568-76.

Mardaryev AN, Ahmed MI, Vlahov NV, Fessing MY, Gill JH, Sharov AA, et al. Micro-RNA-31 controls hair cycle-associated changes in gene expression programs of the skin and hair follicle. FASEB journal 
: official publication of the Federation of American Societies for Experimental Biology 2010;24(10):3869-81.

Paus R, Muller-Rover S, Van Der Veen C, Maurer M, Eichmuller S, Ling G, et al. A comprehensive guide for the recognition and classification of distinct stages of hair follicle morphogenesis. The Journal of investigative dermatology 1999;113(4):523-32.

Pullagura SRN, Buaas B, Gray N, Krening LC, Srivastava A, Braun RE. Functional Redundancy of DICER Cofactors TARBP2 and PRKRA During Murine Embryogenesis Does Not Involve miRNA Biogenesis. Genetics 2018;208(4):1513-22.

Teta M, Choi YS, Okegbe T, Wong G, Tam OH, Chong MM, et al. Inducible deletion of epidermal Dicer and Drosha reveals multiple functions for miRNAs in postnatal skin. Development 2012;139(8):1405-16.

Xu N, Brodin P, Wei T, Meisgen F, Eidsmo L, Nagy N, et al. MiR-125b, a microRNA downregulated in psoriasis, modulates keratinocyte proliferation by targeting FGFR2. The Journal of investigative dermatology 2011;131(7):1521-9.

Yi R, O'Carroll D, Pasolli HA, Zhang Z, Dietrich FS, Tarakhovsky A, et al. Morphogenesis in skin is governed by discrete sets of differentially expressed microRNAs. Nature genetics 2006;38(3):356-62.

Yi R, Pasolli HA, Landthaler M, Hafner M, Ojo T, Sheridan R, et al. DGCR8-dependent microRNA biogenesis is essential for skin development. Proceedings of the National Academy of Sciences of the United States of America 2009;106(2):498-502.

Yu J, Peng H, Ruan Q, Fatima A, Getsios S, Lavker RM. MicroRNA-205 promotes keratinocyte migration via the lipid phosphatase SHIP2. FASEB journal : official publication of the Federation of American Societies for Experimental Biology 2010;24(10):3950-9.

Zhao B, Chen Y, Yang N, Chen Q, Bao Z, Liu M, et al. miR-218-5p regulates skin and hair follicle development through Wnt/beta-catenin signaling pathway by targeting SFRP2. Journal of cellular physiology 2019;234(11):20329-41. 

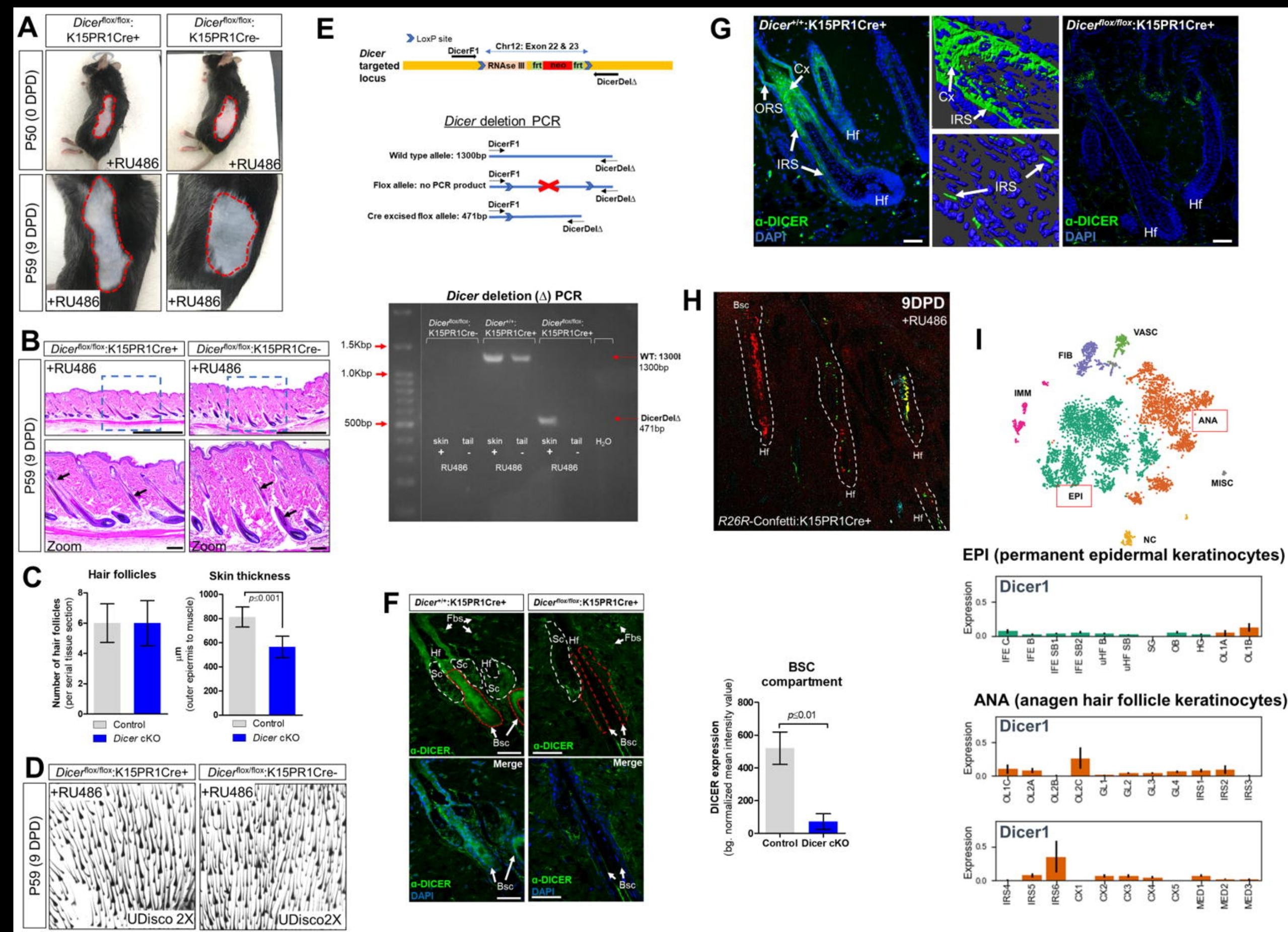

E
G Dicer ${ }^{1 / 4}:$ K15PR1Cre+

F
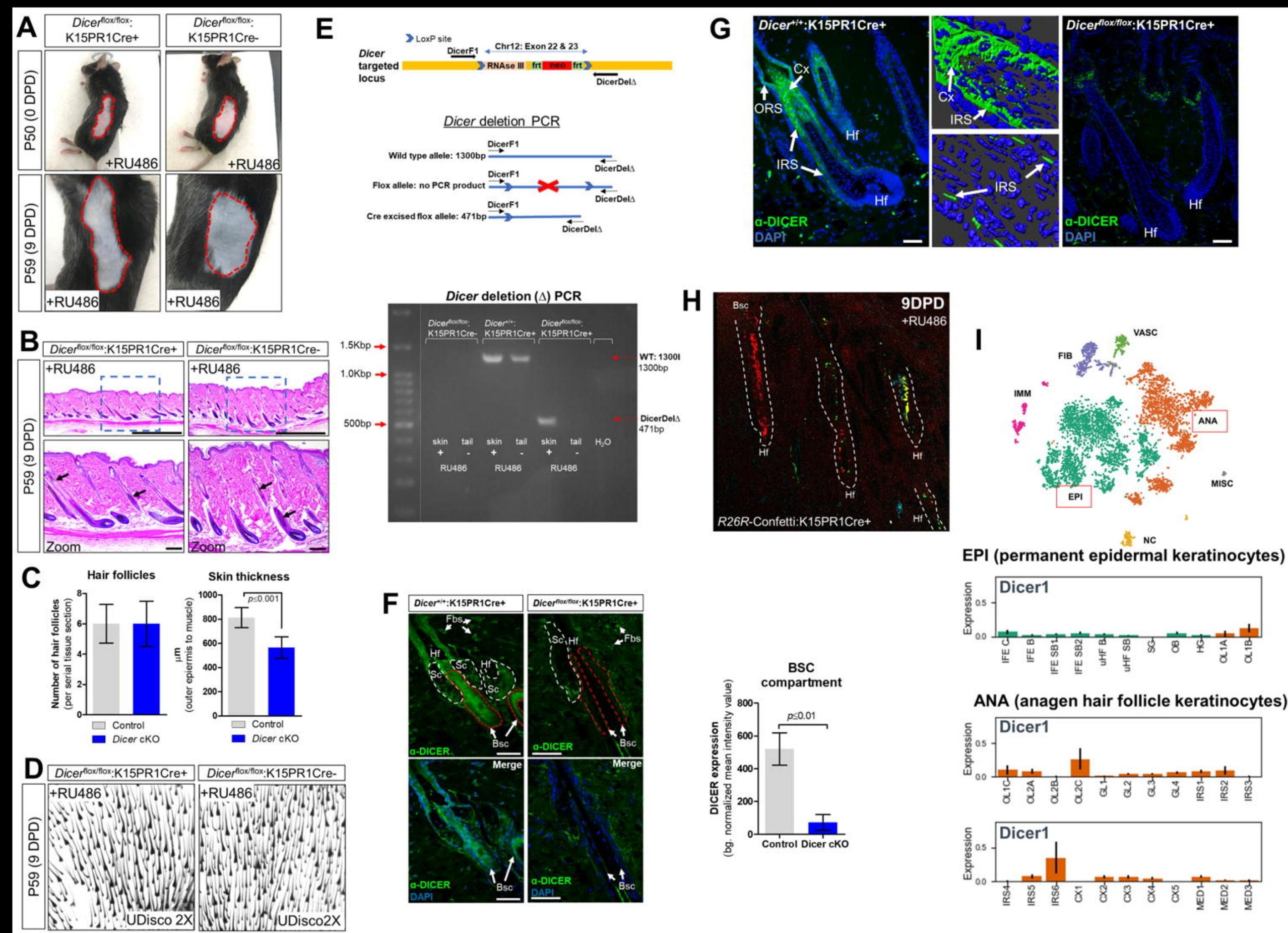

\section{I}

EPI (permanent epidermal keratinocytes)

ANA (anagen hair follicle keratinocytes)

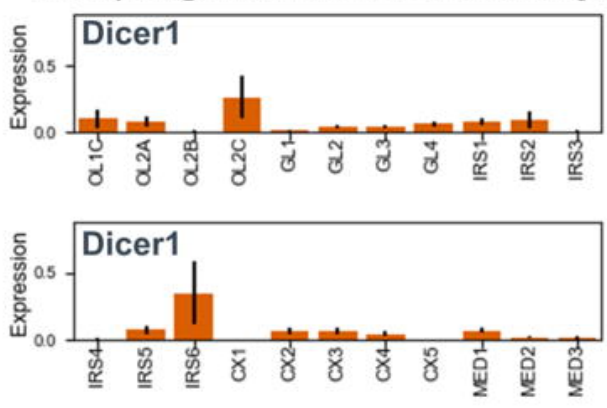
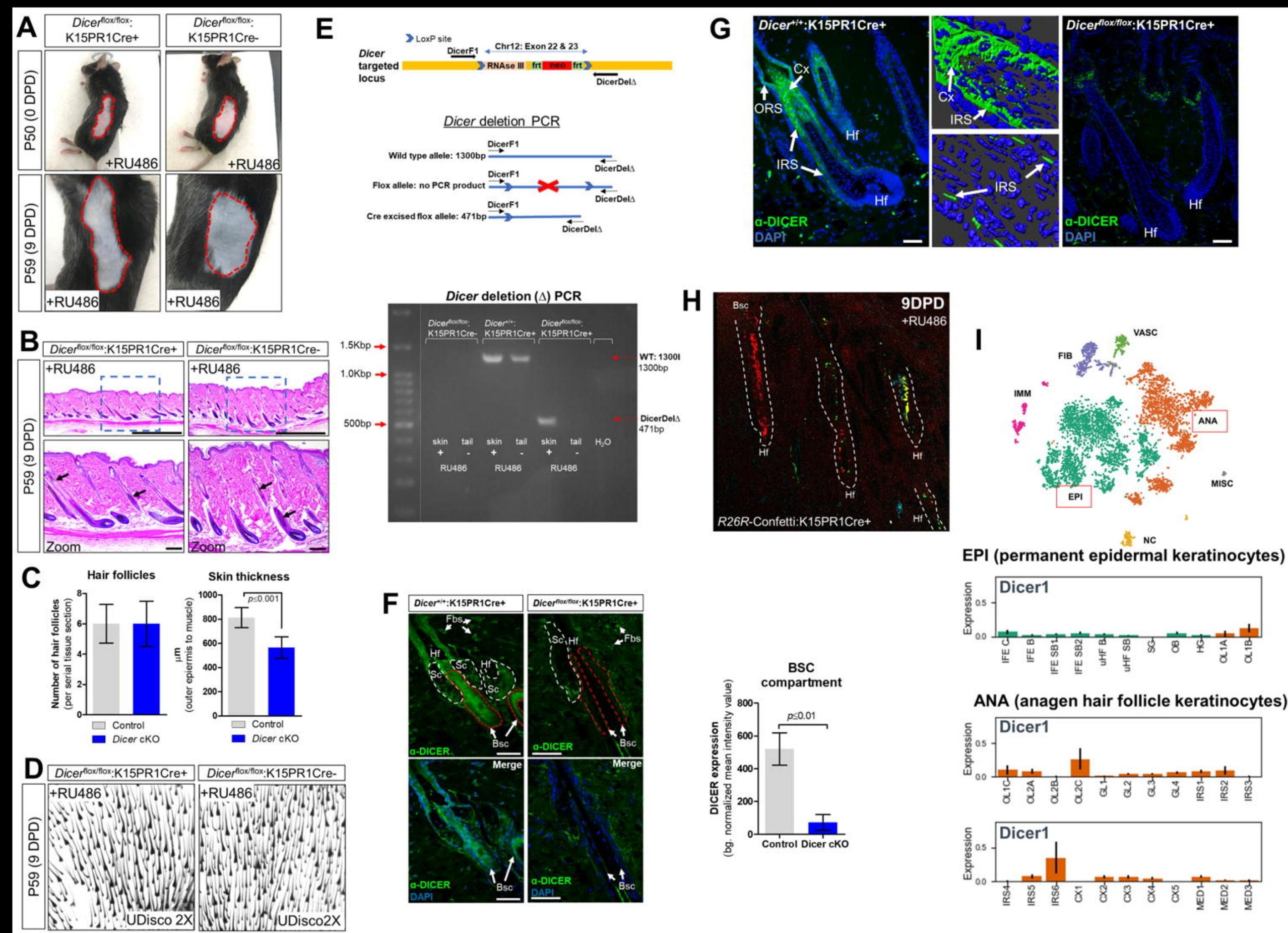

\section{B}

D Dicer floxffox:K15PR1Cre+ Dicerfloxffox:K15PR1Cre-

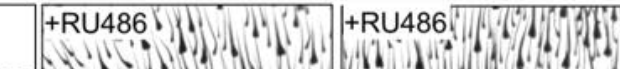
a $A$. a 5 . o 1 .

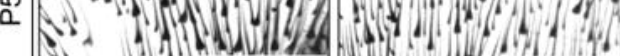

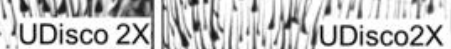

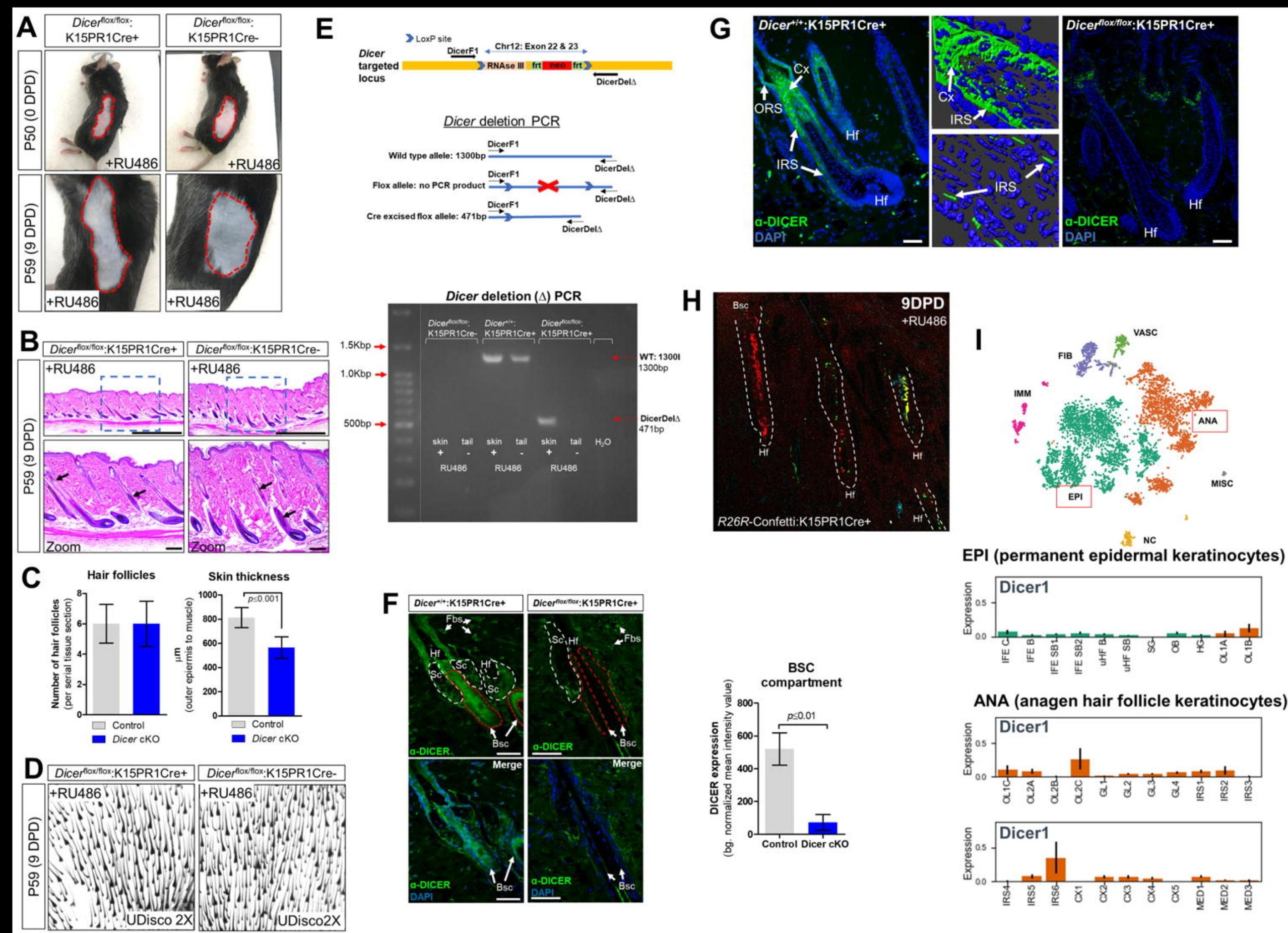

